\title{
Proceedings
}

\section{Digital Image Quality Prediction System ${ }^{\dagger}$}

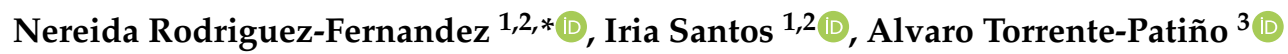 \\ and Adrian Carballal 1,3 (1) \\ 1 CITIC-Research Center of Information and Communication Technologies, University of A Coruña, \\ 15071 A Coruña, Spain; iria.santos@udc.es (I.S.); adrian.carballal@udc.es (A.C.) \\ 2 Department of Computer Science and Information Technologies, Faculty of Communication Science, \\ University of A Coruña, Campus Elviña s/n, 15071 A Coruña, Spain \\ 3 Department of Computer Science and Information Technologies, Faculty of Computer Science, \\ University of A Coruña, Campus Elviña s/n, 15071 A Coruña, Spain; alvaro.torrente@udc.es \\ * Correspondence: nereida.rodriguezf@udc.es \\ + Presented at the 3rd XoveTIC Conference, A Coruña, Spain, 8-9 October 2020.
}

Published: 19 August 2020

\begin{abstract}
A picture is worth a thousand words." Based on this well-known adage, we can say that images are important in our society, and increasingly so. Currently, the Internet is the main channel of socialization and marketing, where we seek to communicate in the most efficient way possible. People receive a large amount of information daily and that is where the need to attract attention with quality content and good presentation arises. Social networks, for example, are becoming more visual every day. Only on Facebook can you see that the success of a publication increases up to $180 \%$ if it is accompanied by an image. That is why it is not surprising that platforms such as Pinterest and Instagram have grown so much, and have positioned themselves thanks to their power to communicate with images. In a world where more and more relationships and transactions are made through computer applications, many decisions are made based on the quality, aesthetic value or impact of digital images. In the present work, a quality prediction system for digital images was developed, trained from the quality perception of a group of humans.
\end{abstract}

Keywords: machine learning; genetic algorithm; quality; image; prediction; dataset

\section{Introduction}

In recent years, significant efforts were applied to the development of successful models and algorithms that can automatically and accurately predict the perceptual quality of two-dimensional (2D) and three-dimensional (3D) digital images and videos. This estimate comes from studies with at least a century of experience, or more if we take into account those developed by Platon and Aristotle, usually from Humanities departments: Psychology, Sociology, Philosophy, Fine Arts, etc. [1]. Different research groups sought to create computer systems capable of learning the aesthetic and quality perception of a group of humans as part of a generative system for uses such as the selection and arrangement of images within a set, even though it is complex to translate this into computer problems. Visual quality refers to the quantification of the perceptual degradation of a visual stimulus due to the presence or absence of distortions. Most of the applications that were developed were designed to treat synthetically distorted images [2]. In this case, unlike other image quality assessment algorithms that use synthetically distorted images [3,4], it was decided to use images with absence of distortion $[5,6]$. Despite the fact that the data collected contained quality and aesthetic results, on this occasion only the quality data were used as they constituted more objective results [7]. 


\section{Materials and Methods}

After analyzing the degree of generalization of some datasets used in automatic image prediction, it was concluded that it was not enough to consider them as a reference in the training of automatic image prediction and classification systems. Taking this into account, a new set of images from the web portal DPChallenge.com was developed in search of greater statistical consistency [7].

The proposed dataset was built following the steps outlined in previous works [8]: obtaining the images on the web portal, filtering those images, organizing them according to their evaluation on the portal and selecting sets with an equal number of images. Subsequently, the quality of the images was evaluated by a group of humans through the Amazon Mechanical Turk platform. This group of humans was made up of 525 inhabitants of the USA (39\% men and 61\% women), aged between 18 and 70. A representation of the images from this dataset is shown in Figure 1.

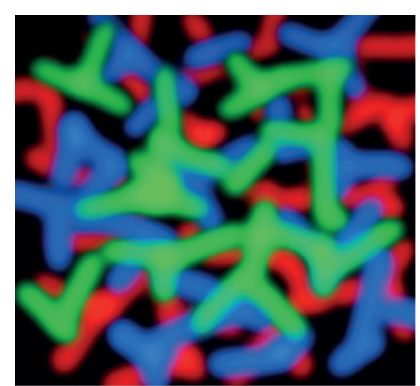

a)

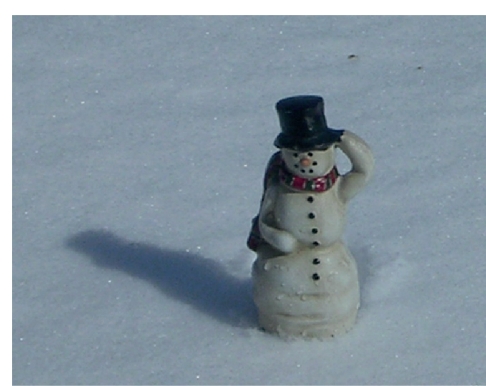

b)

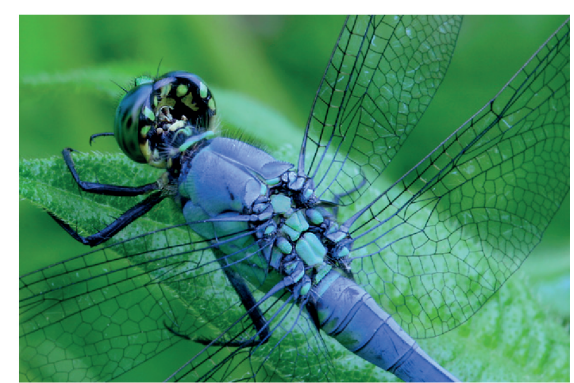

c)

Figure 1. Images of different scoring ranges belonging to the dataset used in this work, evaluated by humans according to their quality. (a) Image with an average score of 2.7 out of a maximum of 10. (b) Image with an average score of 5.7 out of a maximum of 10. (c) Image with an average score of 9.28 out of a maximum of 10 .

With this data, a system was created to predict the quality of digital images with the search engine Correlation by Genetic Search (CGS) [9,10].

\section{Results}

The results obtained during the experimental phase correspond to 50 runs of a 5-fold cross-validation with a training model where $80 \%$ of the set is dedicated to training and the remaining $20 \%$ to testing. As input data, 1024 features of VGG19 were used. The average number of features used in the 50 runs is 114, which has also reached an average Pearson correlation of 0.77 and an average error of 0.15 . Figure 2 shows the distribution of features, Pearson correlation and error of the 50 runs. The absence of a large number of outliers stands out, which provides consistency and validity to the data obtained. In the three cases, the data that is recognized as outlier belongs to the same run. In the case of the error, its greater variability can be observed, with a maximum error of 0.16 and a minimum error of 0.09 . In the case of the features and the Pearson correlation, a much more uniform and concentrated representation is observed, with a very small variability that leads to deduce that the model proposes coherent results in the 50 runs. 


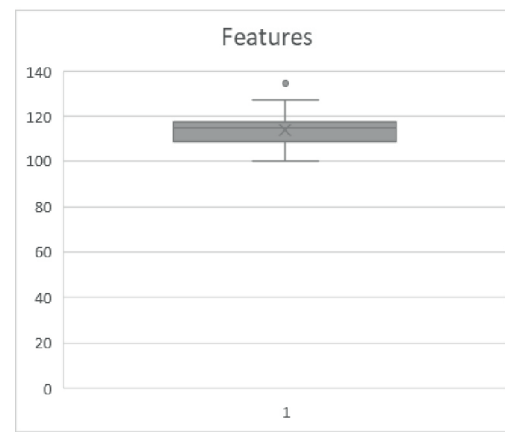

a)

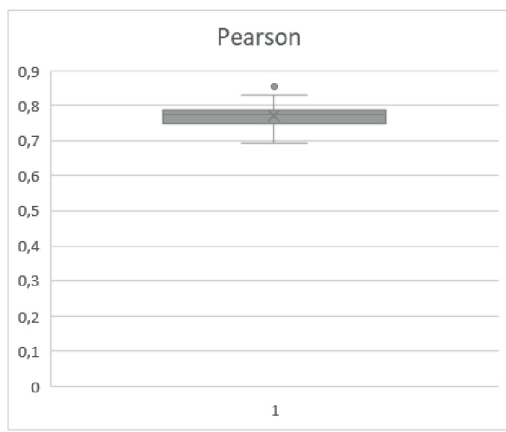

b)

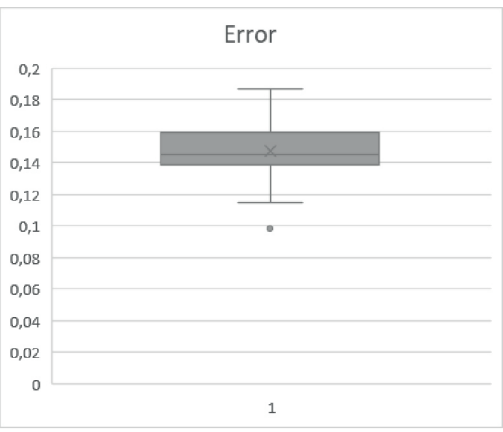

c)

Figure 2. Results obtained in the experiments carried out with CGS. (a) Features used in each of the 50 runs. (b) Pearson correlation of the validation set in each run. (c) Average error obtained in each run.

\section{Conclusions}

This paper focuses on the creation of a digital image quality prediction system from a set of human-evaluated images. The task was tested with a hybrid method for the creation of multiple regression models based on the maximization of the correlation, the CGS method. Thus, an average Pearson correlation of 0.77 in 50 runs with 5 -fold cross-validation was achieved, with a consistent distribution and low variability, which provides better results than other state-of-the-art works such as Nadal et al. [11] or Marin and Leder [12].

Acknowledgments: CITIC, as a Research Centre of the Galician University System, is financed by the Regional Ministry of Education, University and Vocational Training of the Xunta de Galicia through the European Regional Development Fund (ERDF) with 80\%, Operational Programme ERDF Galicia 2014-2020 and the remaining 20\% by the General Secretariat of Universities (Ref. ED431G 2019/01). This work has also been supported by the General Directorate of Culture, Education and University Management of Xunta de Galicia (Ref. ED431D 201716), and Competitive Reference Groups (Ref. ED431C 201849)

\section{References}

1. Maitre, H. A Review of Image Quality Assessment with application to Computational Photography. In Proceedings of the Ninth International Symposium on Multispectral Image Processing and Pattern Recognition, Enshi, China, 31 October-1 November 2015.

2. Celona, L. Learning Quality, Aesthetics, and Facial Attributes for Image Annotation. Tesi di Dottorato, Universitàdegli Studi di Milano-Bicocca, Milan, Italy, 2018.

3. Li, J.; Yan, J.; Deng, D.; Shi, W.; Deng, S. No-reference image quality assessment based on hybrid model. Signal Image Video Process. 2016, 11, doi:10.1007/s11760-016-1048-5.

4. Xu, J.; Ye, P.; Li, Q.; Du, H.; Liu, Y.; Doermann, D. Blind image quality assessment based on high order statistics aggregation. IEEE Trans. Image Process. 2016, 25, 4444-4457.

5. Ghadiyaram, D.; Bovik, A. Automatic quality prediction of authentically distorted pictures. Proc. SPIE 2015, doi:10.1117/2.1201501.005759.

6. Ghadiyaram, P.D. Perceptual Quality Assessment of Real-World Images and Videos. Ph.D. Thesis, The University of Texas, Austin, TX, USA, 2017.

7. Rodriguez-Fernandez, N.; Santos, I.; Torrente, A. Dataset for the Aesthetic Value Automatic Prediction. Proceedings 2019, 21, 31.

8. Carballal, A.; Fernandez-Lozano, C.; Rodriguez-Fernandez, N.; Castro, L.; Santos, A. Avoiding the inherent limitations in datsets used for measuring aesthetics whtn using a machine learning approach. Complexity 2019, 1-12, doi:10.1155/2019/4659809.

9. Carballal, A.; Fernandez-Lozano, C.; Heras, J.; Romero, J. Transfer learning for predicting aesthetics through a novel hyprid machine learning method. Neural Comput. Appl. 2019, 32, 5889-5900. 
10. Pazos Perez, R.I.; Carballal, A.; Rabuñal, J.R.; Mures, O.A.; García-Vidaurrázaga, M.D. Predicting vertical urban growth using genetic evolutionary algorithms in Tokyo's Minato ward. J. Urban Plan. Dev. 2018, 144, 04017024.

11. Nadal, M.; Munar, E.; Marty, G.; Cela-Conde, C. Visualcomplexity and beauty appreciation: Explaining the divergenceof results. Empir. Stud. Arts 2010, 28, 173-191.

12. Marin, M.; Leder, H. Examining complexity acrossdomains: Relating subjective and objective measures of affectiveenvironmental scenes, paintings and music. PLOS ONE 2013, 8, e72412.

(C) 2020 by the authors. Licensee MDPI, Basel, Switzerland. This article is an open access article distributed under the terms and conditions of the Creative Commons Attribution (CC BY) license (http://creativecommons.org/licenses/by/4.0/). 